\title{
Finite-temperature non-equilibrium quasi-continuum analysis of nanovoid growth in copper at low and high strain rates
}

\author{
M. Ponga ${ }^{a}$, M. Ortiz ${ }^{a}$, M.P. Ariza ${ }^{b, *}$ \\ ${ }^{a}$ Division of Engineering and Applied Science, California Institute of Technology, \\ Pasadena, CA 91125, USA \\ ${ }^{b}$ Escuela Técnica Superior de Ingeniería, Universidad de Sevilla, Sevilla 41092, Spain
}

\begin{abstract}
We study dynamic nanovoid growth in copper single crystals under prescribed volumetric strain rates ranging from moderate $\left(\dot{\epsilon}=10^{5} \mathrm{~s}^{-1}\right)$ to high $\left(\dot{\epsilon}=10^{10} s^{-1}\right)$. We gain access to lower strain rates by accounting for thermal vibrations in an entropic sense within the framework of maximum-entropy non-equilibrium statistical mechanics. We additionally account for heat conduction by means of empirical atomic-level kinetic laws. The resulting mean trajectories of the atoms are smooth and can be integrated implicitly using large time steps, greatly in excess of those required by molecular dynamics. We also gain access to large computational cells by means of spatial coarse-graining using the quasicontinuum method. On this basis, we identify a transition, somewhere between $10^{7}$ and $10^{8} \mathrm{~s}^{-1}$, between two regimes: a quasistatic regime characterized by nearly isothermal behavior and low dislocation velocities; and a dynamic regime characterized by nearly adiabatic conditions and high dislocation velocities. We also elucidate the precise mechanisms underlying dislocation emission from the nanovoids during cavitation. We additionally investigate the sensitivity of the results of the analysis to the choice of interatomic potential by comparing two EAM-type potentials.
\end{abstract}

Keywords:

Non-equilibrium statistical thermodynamics, Meanfield theory, Void

\footnotetext{
*Corresponding author

Email addresses: mponga@caltech.edu (M. Ponga), ortiz@caltech.edu (M. Ortiz), mpariza@us.es (M.P. Ariza)
} 
growth, Nanovoids, Finite temperature

PACS: 02.70.-c, 46.15.-x, 61.72.-y, 62.50.-p, 62.20.F-

\section{Introduction}

The mechanisms of void nucleation, growth and coalescence are thought to mediate ductile failure and spallation of metals and, in that connection, have been the subject of extensive study. Under shock loading conditions, voids may nucleate at the nanoscale, e. g., by vacancy diffusion and aggregation (Reina et al., 2011). The resulting nanovoids subsequently grow by the emission of discrete lattice dislocations through a variety of intricate dislocation reaction paths (Marian et al., 2004, 2005; Meyers et al., 2009). At extremely high strain rates, nanovoid growth can be effectively simulated using molecular dynamics (Belak, 2002a; Seppälä et al., 2004b; Dávila et al., 2005; Traiviratana et al., 2007; Rudd, 2009; Bringa et al., 2010b; Lubarda, 2011; Tsuru and Shibutani, 2007). These studies reveal, among other useful insights, that nanovoids indeed cavitate upon the attainment of a critical stress or strain through the emission of shear and/or prismatic dislocation loops, resulting in irreversible void growth.

Despite its appeal, molecular dynamics suffers from a number of essential limitations. Foremost among these is the need to resolve every single thermal vibration of each atom in the computational sample, which restricts typical time steps to the femtosecond range. This restriction immediately places slow or long-term phenomena beyond the reach of molecular dynamics, thereby severely curtailing its scope. In particular, nanovoid growth under strain rates below $\sim 10^{7} \mathrm{~s}^{-1}$, which covers the vast majority of practical applications in engineering, cannot be studied by molecular dynamics. Additional difficulties arise in connection with the thermal and thermodynamical aspects of the simulations. Thus, molecular dynamics simulations are often carried out either in the microcanical or canonical ensemble, in the latter case by recourse to thermostating, Langevin dynamics and other techniques (cf., Andersen (1980); Nosé (1984); Hoover (1985); Hünenberger (2005); Bussi and Parrinello (2008)). However, these computational devices are ad hoc and often unrealistic and compromise the fidelity and predictiveness of the simulations. Not unrelated are the facts that molecular dynamics simulations tend to be noisy, which compounds the interpretation of results, especially 
as regards mesoscale phenomena such as the establishment and evolution of temperature fields, the role of heat transport and others. In particular, atomic-level noise in molecular dynamics simulations obscures lattice-level mechanisms such as dislocation reactions, especially at high strain rates. Finally, the study of defects at realistic concentrations, which are often in the parts per million, requires consideration of extremely large computational cells. Under these conditions, the explicit treatment of every atom in the cell is exceedingly wasteful, as only the cooperative behavior of large numbers of atoms - and not the behavior of individual ones - is of interest. This situation is strongly suggestive of the use of coarse-graining techniques, such as the quasicontinuum method (Tadmor et al., 1996), in order to weed out redundant and inconsequential degrees of freedom.

In this study, we overcome these difficulties by means of the finite-temperature quasicontinuum method (HotQC) of Kulkarni et al.(Kulkarni et al., 2008; Ariza et al., 2012; Ponga et al., 2012; Venturini et al., 2014). The aim of HotQC is to account for thermal effects, including atomic-level heat conduction, without the need for tracking every thermal vibration of the atoms. The approach is based on Jayne's maximum entropy principle (Jaynes, 1957a,b), which enables the statistical treatment of systems away from equilibrium. By maximizing a suitable constrained entropy, effective thermodynamic potentials are identified which characterize the instantaneous state of a system and supply driving forces for its evolution. However, unlike conventional equilibrium thermodynamic potentials such as the free energy, the new thermodynamic potentials are well-defined away from equilibrium, e. g., in the presence of strong temperature gradients. Under such conditions, HotQC characterizes the evolution of the system by means of empirical kinetic equations of the Onsager type (Onsager, 1931a,b; De Groot and Mazur, 1962). These kinetic relations may be regarded as an atomic-level Fourier law of heat conduction and, similarly to interatomic potentials, are subject to modeling. In practice, the exact evaluation of the requisite non-equilibrium thermodynamic potentials is generally intractable. However, they can be effectively approximated by means of variational meanfield theory and the resulting variational framework provides a convenient basis for the formulation of computationally tractable models, with or without spatial coase-graining. The fidelity of HotQC has been carefully assessed by Kulkarni et al. (2008) and Venturini et al. (2014) by direct comparison with experimental observation, including equilibrium properties of metallic alloys including lattice parameter, linear thermal expansion coefficient, elastic moduli and surface segregation con- 
centration. Venturini et al. (2014) have additionally assessed the fidelity of empirical kinetic relations by means of two test cases: Heat conduction in silicon nanowires and hydrogen storage in palladium. In all cases, the ability of HotQC to properly account for equilibrium properties, structure-dependent transport properties and the long-term behavior of atomic systems over exceedingly long times is remarkable.

In the present work, whereas the thermal vibrations are accounted for in an entropic sense by recourse to max-ent, we account explicitly for microinertia, i. e., the dynamics attendant to void growth at the mesoscale. This consideration of mesoscale dynamics raises fundamental and longstanding issues of double counting for inertia, stability of time-stepping algorithms and phonon trapping resulting from spatial coarse-graining. Remarkably, the entropic treatment of thermal vibrations results in mean atomic trajectories that are slowly varying, or smooth, on the thermal time scale. In particular, the mean atomic trajectories are free of thermal vibrations, with the result that inertia is not double-counted. In addition, the smoothness of the mean atomic trajectories enables the use of implicit time-integration methods, such as Newmark's algorithm (Newmark, 1959), with exceedingly large time steps, in effect opening the way for the analysis of slow processes and the long-term behavior of atomistic systems. Finally, the smoothness of the mean atomic trajectories enables the introduction of spatial coarse-graining, e. g., by means of the quasicontinuum method, without spurious internal reflections and wave trapping at boundaries between regions of varying spatial resolution.

By this means, in the present paper we extend previous studies of dynamic nanovoid growth in copper single crystals (Seppälä et al., 2004a; Dávila et al., 2005; Bringa et al., 2010b) to strain rates greatly below those accessible to molecular dynamics. We specifically study the effect of micro-inertia in nanovoids, with particular focus on mechanisms of dislocation emission, for strain rates ranging from moderate $\left(\dot{\epsilon}=10^{5} \mathrm{~s}^{-1}\right)$ to high $\left(\dot{\epsilon}=10^{10} \mathrm{~s}^{-1}\right)$. Another focus of the study concerns the role and extent of heat generation and conduction during dislocation emission. In this regard, HotQC conveniently affords clarity in the interpretation of calculation results by differentiating sharply between heat and temperature and mesoscopic dynamics. In particular, it supplies well-defined atomic-level temperature fields and their evolution. On this basis, we identify a transition, somewhere between $10^{7}$ and $10^{8} \mathrm{~s}^{-1}$, between two regimes: a quasistatic regime characterized by nearly isothermal behavior and low dislocation velocities; and a dynamic regime 
characterized by nearly adiabatic conditions and high dislocation velocities. We additionally investigate the sensitivity of the results of the analysis to the choice of interatomic potential by comparing two EAM-type potentials by Mishin et al. (2001) and Johnson (1988).

\section{Finite-temperature non-equilibrium model}

For completeness, we provide a brief summary of the HotQC approach that serves as the basis of the present work.

\subsection{Non-equilibrium statistical mechanics}

Following Kulkarni et al. (2008) and Venturini et al. (2014), we specifically consider systems consisting of $N$ identical particles, e. g., atoms or molecules. An extension to systems comprised of particles of different species may be found in Venturini et al. (2014). We assume that the statistics of the system obeys Jaynes' principle of maximum entropy (Jaynes, 1957a,b) (see also Zubarev (1974); Callen (1985)). This principle postulates that the probability density function $\rho(\{\boldsymbol{q}\},\{\boldsymbol{p}\})$, characterizing the probability of finding the system in a state $(\{\boldsymbol{q}\},\{\boldsymbol{p}\})$, maximizes the information-theoretical entropy

$$
\mathcal{S}[\rho]=-k_{B}\langle\log \rho\rangle,
$$

among all probability measures consistent with the constraints on the system. In (2.1) and subsequently, $k_{B}$ denotes Bolzmann's constant and $\langle A\rangle$ indicates the expected value of $A$. We specifically consider systems consisting of distinguishable particles whose Hamiltonians have the additive structure

$$
H=\sum_{i=1}^{N} h_{i},
$$

where $h_{i}$ is the local Hamiltonian of particle $i$. Note, however, that each local hamiltonian $h_{i}$ is allowed to depend on the positions, momenta, and species of all the particles in the system. Suppose that the expected particle positions, momenta and energies, namely,

$$
\left\langle\boldsymbol{q}_{i}\right\rangle=\overline{\boldsymbol{q}}_{i}, \quad\left\langle\boldsymbol{p}_{i}\right\rangle=\overline{\boldsymbol{p}}_{i}, \quad\left\langle h_{i}\right\rangle=e_{i},
$$

$i=1, \ldots, N$, respectively, are known. It should be noted that, unlike the classical equilibrium framework where only global constraints are enforced, 
these constraints are now local. Maximizing (2.1) among probability measures results, after a trite calculation, in

$$
\rho=\frac{1}{Z} \mathrm{e}^{-\{\beta\}^{T}\{h\}},
$$

where

$$
Z(\{\overline{\boldsymbol{q}}\},\{\overline{\boldsymbol{p}}\},\{\beta\})=\frac{1}{h^{3 N}} \int_{\Gamma(\{\overline{\boldsymbol{q}}\},\{\overline{\boldsymbol{p}}\})} \mathrm{e}^{-\{\beta\}^{T}\{h\}} d q d p,
$$

and $\{\boldsymbol{\beta}\}$ are Lagrange multipliers. In (2.5) and subsequently, $\Gamma(\{\overline{\boldsymbol{q}}\},\{\overline{\boldsymbol{p}}\})$ is the affine subspace of $\left(\mathbb{R}^{3} \times \mathbb{R}^{3}\right)^{N}$ such that $\langle\boldsymbol{q}\rangle=\{\overline{\boldsymbol{q}}\}$ and $\langle\boldsymbol{p}\rangle=\{\overline{\boldsymbol{p}}\}$, $h$ is Planck's constant and $h^{-3 N}$ supplies the natural unit of phase volume for systems of distinguishable particles (cf., e. g., Hill (1987); Girifalco (2000)). By analogy to equilibrium statistical mechanics, we may interpret (2.4) and (2.5) as non-equilibrium generalizations of the Gibbs canonical probability density function and the canonical partition function, respectively. In addition, by analogy with equilibrium statistical mechanics, we may interpret

$$
\theta_{i}=\frac{1}{k_{B} \beta_{i}}
$$

as the particle absolute temperature. We emphasize that, unlike equilibrium statistical mechanics, the temperature field need not be uniform and may vary from particle to particle when the system is away from equilibrium. In addition, the canonical free entropy

$$
S(\{\overline{\boldsymbol{q}}\},\{\overline{\boldsymbol{p}}\},\{\beta\})=k_{B} \log Z,
$$

defines the relations

$$
\begin{aligned}
& \frac{d \overline{\boldsymbol{p}}_{i}}{d t}=-\frac{1}{k_{B}} \frac{\partial^{2} S}{\partial \beta_{i} \partial \overline{\boldsymbol{q}}_{i}}, \\
& \frac{d \overline{\boldsymbol{q}}_{i}}{d t}=\frac{1}{k_{B}} \frac{\partial^{2} S}{\partial \beta_{i} \partial \overline{\boldsymbol{p}}_{i}}, \\
& e_{i}=-\frac{1}{k_{B}} \frac{\partial S}{\partial \beta_{i}},
\end{aligned}
$$

which generalize the classical Hamilton's and equilibrium equations to thermodynamic systems away from equilibrium.

Following Kulkarni et al. (2008) and Venturini et al. (2014), we additionally seek to characterize the evolution of the discrete temperature field, 
be it towards equilibrium or in response to external driving, by means of kinetic equations of the Onsager type (Onsager, 1931a,b; De Groot and Mazur, 1962). We begin by examining the balance of energy at the particle level. The balance of energy at particle $i$ takes the form

$$
\dot{e}_{i}=\dot{w}_{i}+\sum_{j \neq i} R_{i j}
$$

where $\dot{w}_{i}$ is the work of the externally applied forces and $R_{i j}=-R_{j i}$ is the discrete heat flux from particle $j$ to $i$. Venturini et al. (2014) have formulated discrete kinetic framework by mirroring the classical ColemanNoll method of continuum thermodynamics (Coleman and Noll, 1963), with the aim of deriving a dissipation inequality expressed in terms of discrete thermodynamic fluxes and forces. They find that the entropy production rate attendant to a particle pair is

$$
\Sigma_{i j}=P_{i j} R_{i j}
$$

where

$$
P_{i j}=\frac{1}{\theta_{i}}-\frac{1}{\theta_{j}}
$$

defines the discrete thermodynamic force corresponding to the discrete heat flux $R_{i j}$ from particle $j$ to $i$ and. The entropy production identity (2.10) effectively identifies the appropriate form of the thermodynamic flux and force pertaining to heat transfer between atoms. By analogy with Onsager's theory of kinetic relations (De Groot and Mazur, 1962), the local entropy production identity (2.10), Venturini et al. (2014) posit a kinetic law of the general form

$$
R_{i j}=\frac{\partial \psi}{\partial P_{i j}}(\{P\})
$$

where $\psi(\{P\})$ is a discrete kinetic potential. We may regard this kinetic relation as a discrete Fourier law of heat conduction. An extension of the framework to coupled heat and mass transport may be found in Venturini et al. (2014). The discrete energy balance equation (2.9), together with the discrete kinetic relations (2.28) result in a discrete heat equation that governs the evolution of the discrete temperature field.

We emphasize that, in the present approach, the kinetic relations are regarded as empirical relations and, as such, subject to modeling, in the entirely same manner as empirical interatomic potentials are the subject 
of modeling in the field of molecular dynamics. The modeling task may be simplified by recourse to a number of ancillary modeling assumptions, such as the assumption of linear kinetics, i. e., a linear relation between thermodynamic fluxes and driving forces.

\subsection{Meanfield approximation}

Unfortunately, the calculation of the canonical free energy (2.7) in closed form is generally intractable, which raises the need for approximation theory. Venturini et al. (2014) have extended the classical variational meanfield theory to systems described by the proposed non-equilibrium statisticalmechanical framework. The resulting variational framework provides a convenient basis for the formulation of computationally tractable models.

We specifically consider trial Hamiltonians of the form

$$
H_{0}=\sum_{i=1}^{N} h_{0 i}
$$

where $h_{0 i}$ is the local trial Hamiltonian of particle $i$. Suppose that these local trial Hamiltonians are parameterized by fields $\{\overline{\boldsymbol{q}}\}=\left(\overline{\boldsymbol{q}}_{i}\right)_{i=1}^{N},\{\overline{\boldsymbol{p}}\}=\left(\overline{\boldsymbol{p}}_{i}\right)_{i=1}^{N}$ and $\{\omega\}=\left(\omega_{i}\right)_{i=1}^{N}$, representing the mean position and momenta of the particles and some ad hoc collection of meanfield parameters, respectively. The optimal value of the meanfield parameters $\{\omega\}$ follows variationally by minimization of the meanfield entropy

$$
S_{\mathrm{MF}}(\{\overline{\boldsymbol{q}}\},\{\overline{\boldsymbol{p}}\},\{\beta\},\{\omega\})=k_{B}\{\beta\}^{T}\left\{\left\langle h-h_{0}\right\rangle_{0}\right\}-S_{0}
$$

(cf. Venturini et al. (2014), proposition 4.1), where $\langle A\rangle_{0}$ indicates the expected meanfield value of $A$ with respect to the trial Hamiltonian, $S_{0}$ is

$$
S_{0}=k_{B} \log Z_{0}
$$

and

$$
Z_{0}=\frac{1}{h^{3 N}} \int_{\Gamma} \mathrm{e}^{-\{\beta\}^{T}\left\{h_{0}\right\}} d q d p
$$

are the trial canonical free entropy and partition function, respectively. In addition, the evolution of $(\{\overline{\boldsymbol{q}}\},\{\overline{\boldsymbol{p}}\})$ follows from Hamilton's principle. These 
principles jointly result in the meanfield equations of motion

$$
\begin{aligned}
& \frac{d \overline{\boldsymbol{p}}_{i}}{d t}=-\frac{1}{k_{B}} \frac{\partial^{2} S_{\mathrm{MF}}}{\partial \beta_{i} \partial \overline{\boldsymbol{q}}_{i}}, \\
& \frac{d \overline{\boldsymbol{q}}_{i}}{d t}=\frac{1}{k_{B}} \frac{\partial^{2} S_{\mathrm{MF}}}{\partial \beta_{i} \partial \overline{\boldsymbol{p}}_{i}}, \\
& \frac{\partial S_{\mathrm{MF}}}{\partial \omega_{i}}=0 .
\end{aligned}
$$

These equations define a highly coupled system of equations in the unknowns $(\{\overline{\boldsymbol{q}}\},\{\overline{\boldsymbol{p}}\},\{\omega\})$. We may regard the first two of (2.17) as meanfield equations of motion, whereas the third determines the meanfield parameters $\{\omega\}$ that best represent the Hamiltonian $H$ of the system in a variational meanfield sense. The meanfield local equilibrium relations

$$
e_{i}=-\frac{1}{k_{B}} \frac{\partial S_{\mathrm{MF}}}{\partial \beta_{i}}
$$

and the discrete kinetic relations (2.28) finally close the system of equations that govern the evolution of the entire variable set $(\{\overline{\boldsymbol{q}}\},\{\overline{\boldsymbol{p}}\},\{\beta\},\{\omega\})$.

A simple meanfield model for substitutional alloys is obtained by considering local trial Hamiltonians of the form (Kulkarni et al., 2008)

$$
h_{0 i}(\{\boldsymbol{q}\},\{\boldsymbol{p}\})=\frac{1}{2 m_{i}}\left|\boldsymbol{p}_{i}-\overline{\boldsymbol{p}}_{i}\right|^{2}+\frac{m_{i} \omega_{i}^{2}}{2}\left|\boldsymbol{q}_{i}-\overline{\boldsymbol{q}}_{i}\right|^{2} .
$$

Extensions to alloys have been proposed by Venturini et al. (2014). For trial local Hamiltonians of the form (2.19), a straightforward calculation gives

$$
S_{\mathrm{MF}}=\sum_{i=1}^{N} s_{i}
$$

with

$$
s_{i}=-k_{B}\left(\beta_{i}\left\langle h_{i}\right\rangle_{0}+3 \log \left(\hbar \beta_{i} \omega_{i}\right)-3\right) .
$$

Suppose, specifically, that the Hamiltonian of the system is of the form

$$
H(\{\boldsymbol{q}\},\{\boldsymbol{p}\})=\sum_{i=1}^{N}\left(\frac{1}{2} \frac{\left|\boldsymbol{p}_{i}\right|^{2}}{m_{i}}+V_{i}(\{\boldsymbol{q}\})\right),
$$


where $V_{i}(\{\boldsymbol{q}\})$ is an interatomic potential for particle $i$. Then, the particle meanfield entropy (2.21) further reduces to

$$
s_{i}=-k_{B}\left(\frac{\beta_{i}}{2 m_{i}}\left|\overline{\boldsymbol{p}}_{i}\right|^{2}+\beta_{i}\left\langle V_{i}\right\rangle_{0}+3 \log \left(\hbar \beta_{i} \omega_{i}\right)-3\right),
$$

and the meanfield equations (2.17) and (2.18) to

$$
\begin{aligned}
& \dot{\overline{\boldsymbol{q}}}_{i}=\frac{\overline{\boldsymbol{p}}_{i}}{m_{i}}, \\
& \dot{\overline{\boldsymbol{p}}}_{i}=-\frac{\partial}{\partial \overline{\boldsymbol{q}}_{i}} \sum_{j=1}^{N}\left\langle V_{j}\right\rangle_{0}, \\
& \frac{\partial}{\partial \omega_{i}} \sum_{j=1}^{N} \beta_{j}\left\langle V_{j}\right\rangle_{0}+\frac{3}{\omega_{i}}=0, \\
& e_{i}=\frac{\left|\overline{\boldsymbol{p}}_{i}\right|^{2}}{2 m_{i}}+\left\langle V_{i}\right\rangle_{0}+\frac{\partial}{\partial \beta_{i}} \sum_{j=1}^{N} \beta_{j}\left\langle V_{j}\right\rangle_{0}+\frac{3}{\beta_{i}} .
\end{aligned}
$$

We note that the calculation of the local effective interatomic potential $\left\langle V_{i}\right\rangle_{0}$ takes place over a local phase subspace of small dimensionality. Since $p_{0}$ is Gaussian, the attendant local phase averages can conveniently be computed by numerical quadrature. In the present work, all phase averages with respect to $p_{0}$ are computed using third-order Gaussian-quadrature rules. Further details regarding the use of Gaussian quadrature for the computation of effective interatomic potentials may be found in Kulkarni et al. (2008).

\subsection{Atomic-level heat conduction}

In calculations, we specifically employ a simple discrete heat transport equation consisting of a finite-element discretization of the heat equation based on a Delaunay triangulation of the atomic positions (Kulkarni et al., 2008). This procedure results in a discrete heat transport equation of the form

$$
\Omega_{i} \dot{e}_{i}=\sum_{j \neq i} \kappa\left(\int \nabla N_{i} \cdot \nabla N_{j} d x\right)\left(\theta_{i}-\theta_{j}\right),
$$

where

$$
\Omega_{i}=\int N_{i} d x
$$


is the atomic volume of atom $i, N_{i}$ is the shape function of atom $i$ and $\kappa$ is a thermal conductivity with units of [power] [length $]^{2} /[$ temperature]. Comparing (2.25) and (2.9) affords the identification

$$
R_{i j}=\frac{\kappa}{\Omega_{i}}\left(\int \nabla N_{i} \cdot \nabla N_{j} d x\right)\left(\theta_{i}-\theta_{j}\right),
$$

for the discrete heat fluxes, which in turn corresponds to an assumption of linear kinetics, namely,

$$
R_{i j}=A_{i j}\left(\frac{1}{\theta_{i}}-\frac{1}{\theta_{j}}\right),
$$

with discrete transport coefficients

$$
A_{i j}=-\frac{\kappa \theta_{i} \theta_{j}}{\Omega_{i}} \int \nabla N_{i} \cdot \nabla N_{j} d x .
$$

Evidently, this simple kinetic model contains one single adjustable parameter, namely, the thermal conductivity $\kappa$. This parameter can in turn be calibrated simply from experimentally measured macroscopic thermal conductivity data.

Eqs. (2.24), jointly with (2.25) define a close system of equations of evolution that can be integrated in time to determine the evolution of the mean position and momenta fields $(\overline{\boldsymbol{q}}, \overline{\boldsymbol{p}})$, the meanfield frequencies $\{\omega\}$ and the temperature field $\{\theta\}$.

\subsection{Implicit time discretization}

We effect the requisite time discretization of (2.24) by recourse to variational time discretization (Yang et al., 2006; Kulkarni et al., 2008), which adapts implicit time integration of the backward-Euler type to the variational structure inherent to the max-ent and meanfield formalisms. In addition, we integrate the equations of motion (2.24) also implicitly by recourse to Newmark's algorithm (Newmark, 1959).

The rationale for the use of implicit solvers in calculations, and its consequences thereof, merit careful discussion. Thus, the max-ent formulation treats the thermal vibrations of the atoms statistically and as an entropic effect, with the result that the mean max-ent trajectory $(\overline{\boldsymbol{q}}(t), \overline{\boldsymbol{p}}(t))$ of the particles is smooth on the scale of the thermal vibrations. Indeed, if a rogue particle attempted to vibrate on the thermal time scale, a large amount of 
heat would be generated and then diffused according to the local heat equation. This process is dissipative and would result in the rapid damping out of the attempted thermal vibration. The net effect of this damping mechanism is to avoid double-counting of the thermal vibrations of the atoms and, correspondingly, to keep the mean max-ent trajectories smooth. The smoothness of the mean max-ent trajectories additionally permits the use of implicit methods with exceedingly large time steps on the time scale of thermal vibrations, in effect opening the way for the analysis of slow processes and the long-term behavior of thermodynamic systems.

Finally, the smoothness of the max-ent trajectories enables the introduction of spatial coarse-graining, e. g., by means of the quasicontinuum method, without spurious internal reflections and wave trapping at boundaries between regions of different spatial resolution.

\subsection{Quasicontinuum spatial coarse-graining}

The statistical-mechanical approach outlined in the foregoing may be regarded as a scheme for coarse-graining molecular dynamics in time. In many applications, including nanovoid cavitation processes such as considered here, the resulting fields vary slowly away from lattice defects and are, therefore, additionally susceptible of spatial coarse-graining. To this end, we resort to the quasicontinuum method of Kulkarni et al. (2008) (HotQC), which extends the quasicontinuum method of (Tadmor et al., 1996) to theremomechanically coupled problems, including heat conduction.

The chief objective of the quasicontinuum method is to systematically coarsen an atomistic description by the judicious introduction of kinematics constraints (Tadmor et al., 1996; Miller and Tadmor, 2002). These kinematic constraints are designed so as to preserve full atomistic resolution where required and to treat collectively large number of atoms in regions where the fields vary slowly on the scale of the lattice. The specific implementation of the quasicontinuum method employed in this work is due to Knap and Ortiz (2001), as extended to non-equilibrium finite temperature by Kulkarni et al. (2008). The desired degree-of-freedom reduction is achieved by restricting the solution, in a Galerkin sense, to subspaces spanned by finiteelement shape functions based on triangulations of a suitably chosen set of representative atoms. The selection of these atoms is effected adaptively so as to provide full atomistic resolution in the vicinity of lattice defects such as dislocations. Finally, the reduced evolution equations are evaluated using summation rules, akin to quadrature rules in finite elements (Knap and 
Ortiz, 2001). Further details of implementation may be found in Knap and Ortiz (2001) and Kulkarni et al. (2008).

\section{Application to void growth}

We proceed to apply HotQC to the study of void cavitation by dislocation emission in copper single crystals under loading conditions ranging from quasistatic, not accessible to molecular dynamics, to dynamic. We specifically consider a spherical $2.2 \mathrm{~nm}$ void centered in a cube of copper of side length $72 a_{0}$ under triaxial expansion loading conditions. At high strain rates and finite temperature, this configuration has been previously studied using molecular dynamics (MD) (Seppälä et al., 2004a). The computational domain is assumed to be thermally insulated and the strain is applied by means of displacement control at its outer boundary at $0.1 \%$ increments. A macroscopic thermal conductivity of $401 \mathrm{~W} / \mathrm{mK}$ was considered within the simulations. The quasistatic equilibrium configurations of the crystal are determined by means of a nonlinear conjugate-gradient algorithm (Vetterling et al., 2002) or, if convergence is too slow, by means of dynamic relaxation (Underwood, 1983; Oakley and Knight, 1995). In addition, the evolution of the atomic temperature at each deformation step is computed by means of a separate nonlinear conjugate-gradient iteration. In dynamic calculations, the equations of motion are integrated in time by means of the explicit Newmark method (Hughes, 2000).

The stacking-fault energy of $\mathrm{Cu}$ is measured at Carter and Ray (1977), $45 \mathrm{~mJ} / \mathrm{m}^{2}$ (cf., also Ogata et al. (2002) for ab initio calculations), which is relatively low among FCC metals. This low value favors dissociation of dislocations into Shockley partials with Burgers vector $\langle 112\rangle$ on planes $\{111\}$ (Hirth and Lothe, 1991), a mechanism that may be expected to strongly influence the dislocation structures attendant to cavitating voids. Based on these considerations, in calculations we use the interatomic potential of Mishin et al. (2001), which predicts an accurate stacking-fault energy of 44.6 $\mathrm{mJ} / \mathrm{m}^{2}$ for copper. From Fig. 3.1 we observe some discrepancies arising when comparing the virial stress curves obtained using the HotQC method with the MD results (Seppälä et al., 2004). The quantitative differences suggest that the inertia effects, which are not embraced in the quasistatic HotQC results are important at a strain rate of $\dot{\varepsilon}=10^{10} \mathrm{~s}^{-1}$ and therefore should not be disregarded. However, despite this fact, both curves present a good qualitative agreement. 


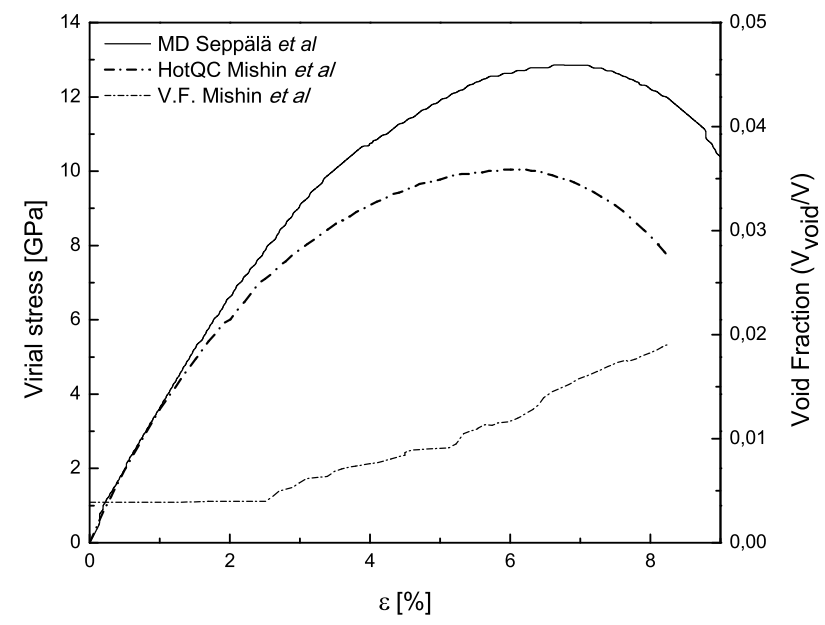

Figure 3.1: Comparison of virial stress and void volume fraction vs engineering strain simulated with HotQC using the EAM-Mishin potential (Mishin et al., 2001) and MD results obtained by Seppälä et al. (2004a) for the same simulation sample $\left(72 a_{0}^{3}\right.$ and void radius $2.2 \mathrm{~nm})$. The triaxial expansion is carried out at $T_{0}=300 \mathrm{~K}$ and $\dot{\varepsilon}=10^{10} \mathrm{~s}^{-1}$.

\subsection{The quasistatic-to-dynamic transition}

We begin by ascertaining the importance of microinertia as a rate-limiting effect in cavitating voids. In particular, we aim to determine the critical strain rate $\dot{\varepsilon}_{c}$ below which the process is ostensibly quasistatic and microinertia can be safely neglected. Ariza et al. (2012) have estimated the critical strain rate separating the quasistatic and dynamic regimes on the basis of analytical expressions for the dynamic expansion of a spherical void in a rigid-plastic material (Ortiz and Molinari, 1992). Here, instead, we proceed to determine the critical strain rate directly by comparing the results of test quasistatic and dynamic calculations at different volumetric strain rates. We find that the quasistatic and dynamic responses of the sample, e. g., measured by the evolution in time of the virial pressure or the void volume fraction, are ostensibly identical up to strain rates of the order of $\dot{\varepsilon}_{c} \sim 10^{7} \mathrm{~s}^{-1}$, Fig. 3.2, and gradually diverge at higher strain rates. For instance, at a strain rate of $10^{10} \mathrm{~s}^{-1}$ the dynamic peak virial pressure is of the order of $30 \%$ larger than the quasistatic peak virial pressure, Fig. 3.1.

It should be carefully noted that, even though microinertia ceases to be important under quasistatic conditions, i. e., at sub-critical strain rates below $\dot{\varepsilon}_{c}$, the material still possesses an intrinsic time scale set by heat conduction, 


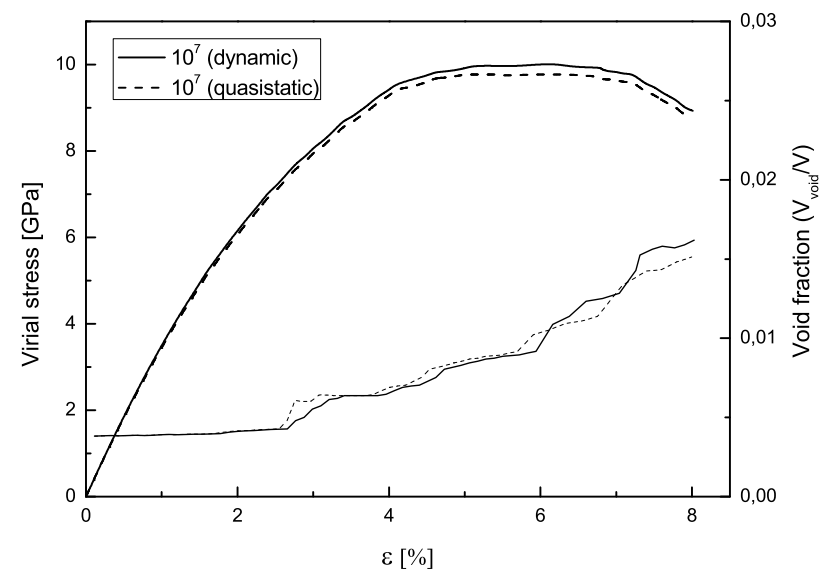

Figure 3.2: Comparison of dynamic and quasistatic HotQC results of the virial stress and normalized void volume under triaxial expansion at a strain rate of $10^{7} \mathrm{~s}^{-1}$ and initial temperature $T_{0}=300 \mathrm{~K}$.

eq. 2.25. This intrinsic time scale introduces rate-dependency in the response of the void and, in particular, discriminates between slow processes, which tend to be isothermal, and fast processes, which tend to be adiabatic.

\subsection{Strain-rate sensitivity in the quasistatic and dynamic regimes}

Fig. 3.3 shows the evolution of the virial stress and void volume fraction at volumetric strain rates in the range $\dot{\varepsilon}=10^{5}$ to $10^{10} \mathrm{~s}^{-1}$ and at an initial temperature $T_{0}=300 \mathrm{~K}$. As expected, the peak pressure and the corresponding volumetric strain are increasing functions of the prescribed volumetric strain rate. The influence of inertia effects on void growth is most clearly discernible from the void volume fraction curves. Thus, within the quasistatic regime of strain rates $\dot{\varepsilon}=10^{5}$ to $10^{7} \mathrm{~s}^{-1}$, the void cavitates soon after the initial elastic stage at volumetric strains of the order of $2 \%$. By contrast, in the dynamic regime of higher strain rates cavitation is much delayed by microinertia to volumetric strains of the order of $6 \%$.

Following the onset of cavitation, dislocations are rapidly emitted from the void surface, with an attendant sharp increase in void growth rate, Fig. 3.5. We find that the prescribed volumetric strain rate also has a strong influence on the rate of dislocation emission. This effect may be quantified by measuring dislocation lengths at different times during the loading processes. Table 1 tabulates the radial distance from the centre of the void to 


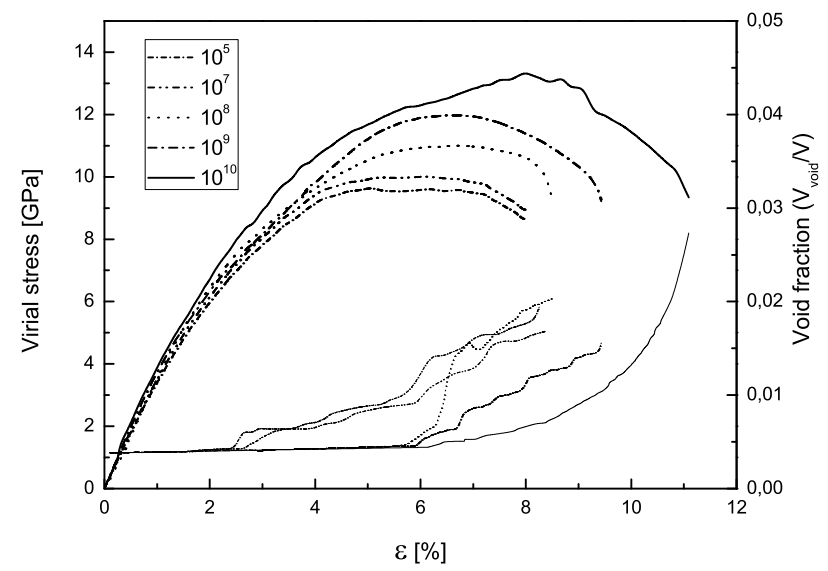

Figure 3.3: Computed virial stress and normalized void volume expansion versus engineering strain $\varepsilon$ of the sample. The triaxial expansion is carried out at strain rates ranging up to $10^{10} \mathrm{~s}^{-1}$ and initial temperature $T_{0}=300 \mathrm{~K}$.

the furthest prismatic loop, Fig. 3.5, corresponding to Shockey partial type dislocations $(1 / 6\langle 211\rangle\{111\})$, at different strain levels over the entire range of strain rates under consideration. These data again reveal a clear retardation of dislocation emission to higher volumetric strains with increasing prescribed volumetric strain rate.

Molecular dynamics simulations for copper (Tsuzuki et al., 2008) deformed in shear have shown the existence of three regimes of dislocation velocity: subsonic, transonic and supersonic. Also, Dávila et al. (2005) have reported the existence of dislocations moving at very high velocities (4.4 $\mathrm{km} / \mathrm{s}$ ) following the interaction of a shock wave with a nanovoid of $2 \mathrm{~nm}$ in radius. Bringa et al. (2010b) have obtained dislocation loop velocities also in copper containing nanovoids as a function of loading orientation.

In the present work, we have likewise endeavored to predict dislocation velocities as a function of applied strain rate. In order to determine representative values of dislocation velocities and their dependence on applied strain rate, we have followed the movement of partial dislocation loops along their characteristic directions (Figs. 3.5 and 3.4) and averaged out the corresponding velocities (Fig. 3.6). The results summarized in table 2 for applied strain rates in the range of $10^{8}$ to $10^{10} \mathrm{~s}^{-1}$ are commensurate with previous observations by Germann et al. $(2005)\left(1.4 \mathrm{~km} / \mathrm{s}\right.$ at a strain rate of $\left.10^{9} \mathrm{~s}^{-1}\right)$ and Bringa et al. (2010b) $(\sim 1 \mathrm{~km} / \mathrm{s})$. By contrast, below the quasistatic-to- 


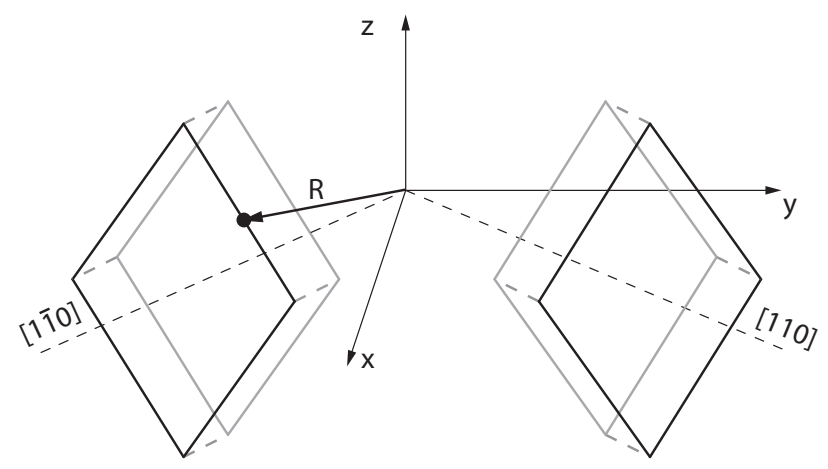

Figure 3.4: Schematic of radial distance from the void center to the first prismatic dislocation loop moving along their characteristic directions [110].

dynamic transition we obtain remarkably low dislocation velocities, namely, 21.25 and $0.07 \mathrm{~m} / \mathrm{s}$ at strain rates of $10^{7}$ and $10^{5} \mathrm{~s}^{-1}$, respectively. This sharply differing behavior further highlights the strongly nonlinear dependence of dislocation velocity on applied strain rate and, in particular, the existence of a sharp transition separating quasistatic and dynamic behavior. It also evinces the difficulties inherent to extrapolating high strain-rate properties down to the quasistatic range.

\subsection{Dislocation structures}

The dislocation structures that mediate nanovoid cavitation, and their evolution, are of particular physical interest. We restrict attention to dislocation structures arising at an applied strain rate of $\dot{\varepsilon}=10^{10} \mathrm{~s}^{-1}$ and an initial temperature of $300 \mathrm{~K}$, as the dislocation structures at other strain rates are ostensibly similar. We have employed the Dislocation Extracting Algorithm (DXA) (Stukowski and Albe, 2010) for purposes of interpreting the results of the calculations and identifying the character of the resulting dislocations. The virial stress vs. strain relation for the latter simulation is represented by the solid-line curve in Fig. 3.2. It is observed from this curve that, following an initial elastic regime, the response of the crystal softens gradually causing the pressure to go through a maximum. The first dislocation structures emerge immediately following the first yield point at a tensile pressure of $12.60 \mathrm{GPa}$, corresponding to a strain of $\varepsilon=6.7 \%$, which may be regarded as the critical tensile pressure for the initiation of plastic deformation. At this point of the process, the maximum shear stress is attained at the inter- 


\begin{tabular}{|c|c|c|c|c|c|}
\hline$\varepsilon[\%]$ & $10^{5} \mathrm{~s}^{-1}$ & $10^{7} \mathrm{~s}^{-1}$ & $10^{8} \mathrm{~s}^{-1}$ & $10^{9} \mathrm{~s}^{-1}$ & $10^{10} \mathrm{~s}^{-1}$ \\
\hline 3.0 & 3.02 & - & - & - & - \\
\hline 4.0 & 5.15 & 1.08 & - & - & - \\
\hline 5.0 & 5.80 & 2.9 & - & - & - \\
\hline 6.0 & 6.73 & 4.7 & - & - & - \\
\hline 6.25 & 6.84 & 5.44 & 2.6 & - & - \\
\hline 6.5 & 6.97 & 6.15 & 4.8 & 2.9 & 3.4 \\
\hline 6.75 & 7.12 & 6.88 & 7.3 & 3.2 & 4.35 \\
\hline 7.0 & 7.22 & 7.56 & 9.7 & 4.5 & 5.85 \\
\hline 8.0 & 8.02 & 8.4 & 10.5 & 5.7 & 6.63 \\
\hline 9.0 & - & - & - & 7.0 & 7.35 \\
\hline 10.0 & - & - & - & 8.22 & 8.15 \\
\hline 11.0 & - & - & - & - & 8.95 \\
\hline
\end{tabular}

Table 1: Dislocation lengths (nm) measured from the center of the computational cell to atoms located on the edge of prismatic dislocation loops (Figs. 3.4 and 3.5), at different times during triaxial loading for strain rates ranging from $10^{5}$ to $10^{10} \mathrm{~s}^{-1}$.

\begin{tabular}{|c|c|c|c|}
\hline$\dot{\varepsilon}\left(\mathrm{s}^{-1}\right)$ & $10^{8}$ & $10^{9}$ & $10^{10}$ \\
\hline velocity $(\mathrm{km} / \mathrm{s})$ & 1.15 & 1.35 & 1.85 \\
\hline
\end{tabular}

Table 2: Dislocation velocities at different strain rates. 


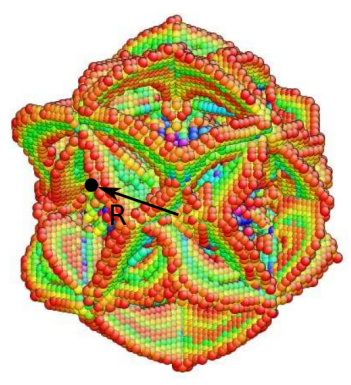

(a)

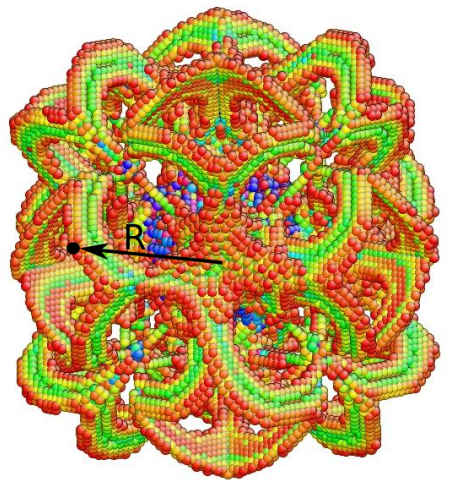

(b)

Figure 3.5: Vector position $R$ from the center of the computational cell to one of the first prismatic dislocation loops at two different times (a) arbitrary time step $t_{0}$, (b) arbitrary time step $t_{1}$.

sections between each of eight $\{111\}$ planes and the void surface. Upon the attainment of the critical tensile pressure, leading Shockley partial dislocations are emitted from the void surface (labelled with letter B in Fig. 3.7a), glide and combine with dislocations moving on another intersecting $\{111\}$ plane. In this manner, Lomer-Cottrell dislocations (labelled 'A' in Fig. 3.7a) are formed along $\langle 110\rangle$ directions, resulting in an octahedral arrangement of the surface atoms. This finding is in keeping with observations of void evolution in FCC metals during spall experiments (Stevens et al., 1972) and molecular dynamics simulations (Belak, 2002b; Seppälä et al., 2004a; Meyers et al., 2009).

The octahedral structure that arises at first yield (Fig. 3.7a) is very stable and temporarily locks the movement of the leading Shockley partials. As the applied strain increases, secondary shear loops grow on $\{111\}$ planes and along the edges of the octahedron. These additional Shockley partial dislocations are highly mobile and glide away from the void. Fig. 3.7b shows the initial stage of emission of the latter shear loops, which eventually intersect and form once again Lomer-Cottrell locks according to the reaction

$$
1 / 6[1 \overline{2} 1]+1 / 6[\overline{2} 1 \overline{1}] \rightarrow 1 / 6[\overline{11} 0] .
$$

Fig. 3.8 shows the Lomer-Cottrell junctions formed surrounding the void, 


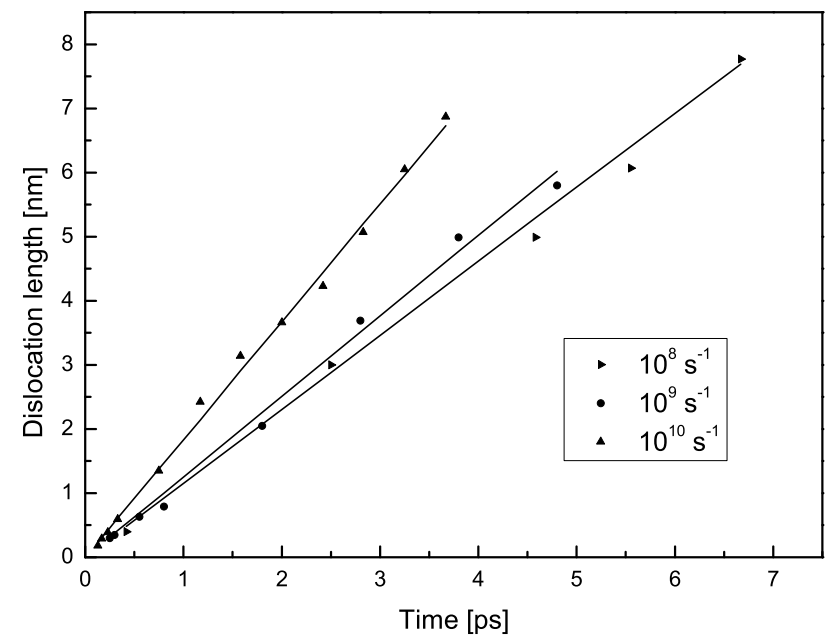

Figure 3.6: Dislocation lengths as function of time (scatter plots) and average velocities of partial dislocation loops (solid lines) at different strain rates.

where we use Common Neighbor Analysis (CNA) (Honeycutt and Andersen, 1987) for purposes of visualization and only atoms with 38 or fewer neighbors are shown. Fig. 3.8 also shows that the $1 / 6\langle\overline{11} 0\rangle$ stair-rod sessile dislocations along the intersection of pairs of $\{111\}$ planes form a closed loop with rhomboid shape and centered on $\langle 110\rangle$ directions, which provides the basis for the subsequent emission of Prismatic Dislocation Loops (PDLs).

Fig. 3.9 shows a series of six snapshots of the nucleation, growth and motion of PDLs as observed in our simulations. This process starts with the emission of secondary Shockley partials along $\langle 110\rangle$ directions (Fig. 3.9a), which upon intersection form stair-rod dislocations (Fig. 3.8). With increasing deformation, new dislocation shear loops grow along the perimeter of the rhombus at a strain of $\sim 8 \%$. Fig. $3.9 \mathrm{~b}$ shows the first two loops moving on the planes intersecting at one vertex of the rhombus. The other two loops lying on the remaining two planes are not clearly visible yet at this strain. The early stages of intersection of the four shear loops is shown in Fig. 3.9c. Completely closed PDLs discernibly detached and moving away from the void surface are evident in Fig. 3.9d. Henceforth, PDLs move away in $\langle 110\rangle$ directions along glide cylinders of rhomboidal section (Figs. 3.9e and 3.9f). These structures and their evolution have been comprehensively described and discussed at length (Hirth and Lothe, 1991; Hull and Bacon, 


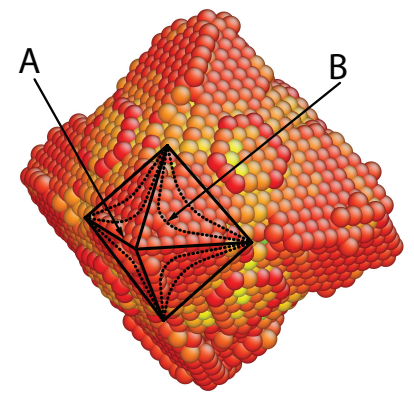

(a)

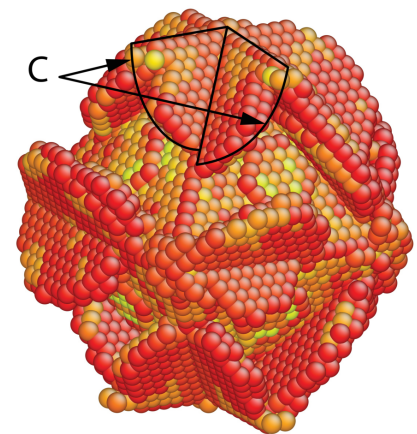

(b)

Figure 3.7: (a) Octahedral structure surrounding the void at $\varepsilon=6.7 \%$. (b) Emission of shear loops along the intersection of $\{111\}$ planes at $\varepsilon=7.25 \%$. In these figures only atoms out of perfect lattice site in FCC structure are shown by using the centrosymmetry analysis.

2001; Bulatov et al., 2010; Bringa et al., 2010a; Rudd, 2009) for crystals of different structures. Finally, above a strain $\varepsilon=11.0 \%$, nucleation of vacancies is observed, eventually leading to material failure. These vacancies nucleate chiefly at intersections between shear loops, reminiscent of the vacancies that arise in spallation tests at shear band intersections (Heller, 2002).

\subsection{Temperature evolution}

Next, we turn to the evolution of the temperature field attendant to the cavitating nanovoids. As already mentioned, cf. Section 2, in the present calculations thermal fluctuations are not tracked explicitly. Instead they are accounted for in an effective manner through the max-ent formalism, with the remaining dynamics governing the time-averaged or mean motion of the atoms. In particular, the motion of the atoms is free of thermal noise, which aids in the identification of deformation mechanisms, including dislocation structures, and generally in the interpretation of the results of the calculations. An additional conceptual benefit of the max-ent paradigm is the introduction of an atomic-level temperature field, not necessarily in equilibrium and evolving according to heat-transport kinetics. Here again, the atomic-level temperature field greatly aids in the elucidation of thermal effects and their coupling to deformation mechanism. 


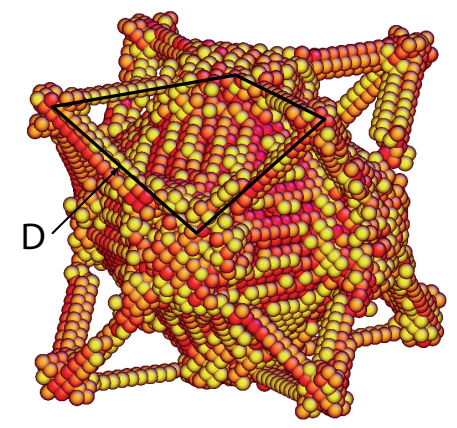

Figure 3.8: Lomer-Cottrell lock with Burgers vector 1/6[110] identified using the Common Neighbor Analysis (CNA) pattern.

Fig. 3.10 shows a closeup of the computed temperature field in the vicinity of the void, before and after dislocation emission. In all cases, the computational domain is assumed to be thermally insulated and, prior to loading, the sample is relaxed at a constant temperature $T_{0}$ by minimizing the mean-field free energy with respect to nodal frequencies $\{\omega\}$ and positions $\{\overline{\boldsymbol{q}}\}$. Fig. 3.11 shows the temperature evolution corresponding to an initial temperature of $300 \mathrm{~K}$ and an applied strain rate of $10^{10} \mathrm{~s}^{-1}$ at three points within the sample: a point on the surface of the void, a second point near the void and a third point far from the void.

Also shown for comparison is a spatially uniform mean temperature obtained from molecular dynamics calculations (Seppälä et al., 2005). It is seen from Fig. 3.11 that the crystal cools down initially due to the thermoelastic effect. Remarkably, the temperature at all sampling points subsequently experiences sudden jumps coincident with the passage of dislocations through the point. These temperature jumps are the result of heat generated by the dislocation motion. Thus, the first temperature jump corresponds to the first set of dislocations emitted from the void surface, whereas subsequent jumps correspond to the passage of prismatic loops. The subsequent decay in time of the temperature recorded at all sampling points, resulting from heat conduction driven by sharp spatial gradients in the temperature field, is also clearly visible in the figure. Analogously, Fig. 3.12 shows the temperature evolution at the same aforementioned three points within the sample when the applied strain rate is lowered to $10^{8} \mathrm{~s}^{-1}$. In general, the calculations 


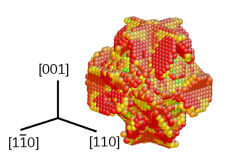

(a)

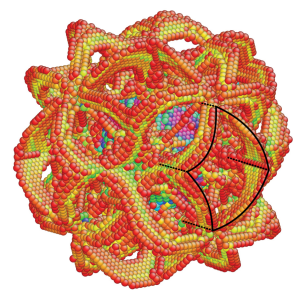

(d)

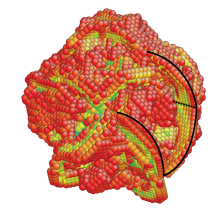

(b)

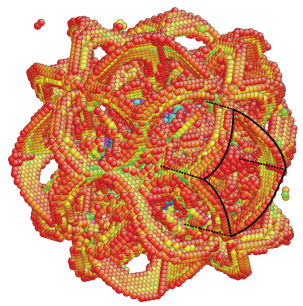

(e)

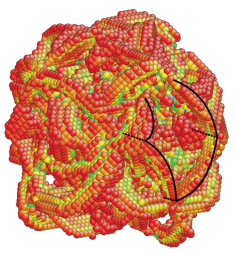

(c)

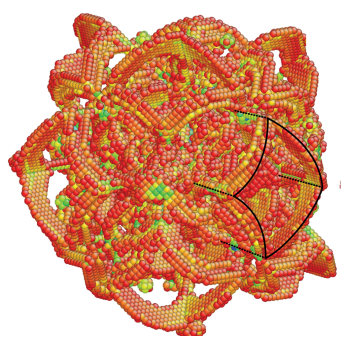

(f)

Figure 3.9: Snapshots embracing the nucleation, motion and interaction of dislocation structures (PDLs) during triaxial expansion with $T_{0}=300 \mathrm{~K}$ and $\dot{\varepsilon}=10^{10} \mathrm{~s}^{-1}$. The snapshots are taken at strain levels: (a) $\varepsilon=7.25 \%$, (b) $\varepsilon=8.0 \%$, (c) $\varepsilon=8.75 \%$, (d) $\varepsilon=9.5 \%$, (e) $\varepsilon=10.25 \%$, (f) $\varepsilon=11.0 \%$.

reveal the emergence of complex temperature fields that are tightly coupled to - and strongly influence - the evolution of the dislocation structures. Here again, the ability of the max-ent paradigm to evince and elucidate this complex thermo-mechanical coupling is noteworthy.

Fig. 3.13 depicts the evolution of the temperature field near the surface of the void at strain rates spanning the range $10^{5}$ to $10^{10} \mathrm{~s}^{-1}$. The initial thermoelastic effect and the temperature jumps upon the passage of dislocations are clearly visible in the figure. Remarkably, the amplitude of the temperature jump, and the strain at which the jump takes place, are observed to increase with applied strain rate. In addition, there is an overall trend towards isothermal response at low applied strain rates and adiabatic response at high applied strain rates. This transition clearly evinces the time dependency and rate sensitivity set in by heat conduction, even under quasistatic conditions.

We have carried out calculations at different initial temperatures ranging 


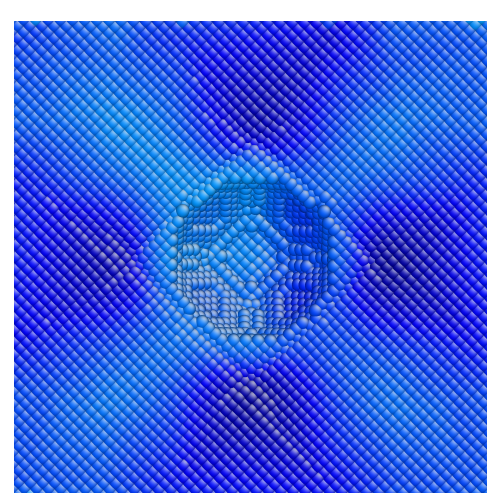

(a)

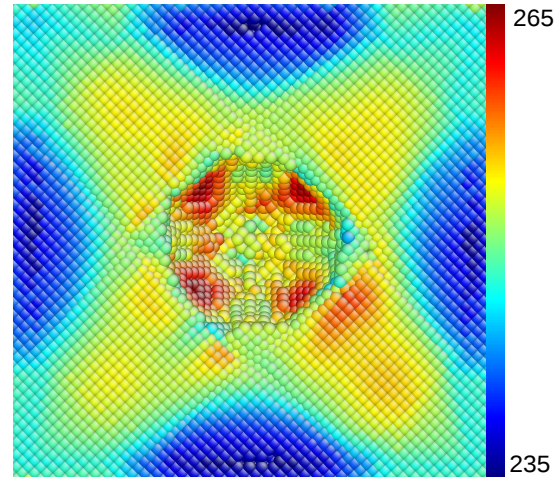

(b)

Figure 3.10: Temperature field in the vicinity of the void (a) before $(\varepsilon=6.6 \%$ ) and (b) after $(\varepsilon=7.3 \%)$ the attainment of the critical strain for dislocation emission $\left(T_{0}=300 \mathrm{~K}\right.$ and $\left.\dot{\varepsilon}=10^{10} \mathrm{~s}^{-1}\right)$.

from 150 to $600 \mathrm{~K}$ and two extreme strain rates, $10^{5}$ and $10^{10} \mathrm{~s}^{-1}$, with a view to elucidating the effect of initial temperature and applied strain rate on the critical virial tensile pressure for cavitation. As may be seen from Fig. 3.14, the critical tensile pressure increases markedly with strain rate, indicative of strong rate-sensitivity. In addition, the critical tensile pressure is relatively temperature-insensitive, or athermal, at low temperatures and decreases steadily beyond a transition temperature of the order of $250 \mathrm{~K}$, indicative of strong thermal activation. The complex interplay between rate and thermal effects during nanovoid cavitation is particularly noteworthy.

\subsection{Interatomic potentials}

In order to ascertain the sensitivity of void growth calculations to the choice of interatomic potential, we have compared the potential of Mishin et al. (2001) employed in the calculations just described (henceforth referred to as EAM-Mishin) and the potential of Johnson (1988) (henceforth referred to as EAM-Johnson). Salient differences between these potentials include their cut-off radii and the resulting stacking-fault energies $\left(\gamma_{S F}\right)$. Thus, the cut-off radius for EAM-Johnson is 1.35 nearest-neighbor distances, whereas the cut-off radius of EAM-Mishin is 2.0 nearest-neighbor distances. A comparison of the total energy of copper computed from the EAM-Johnson and EAM-Mishin potentials is shown in Fig. 3.15. Whereas the differences be- 


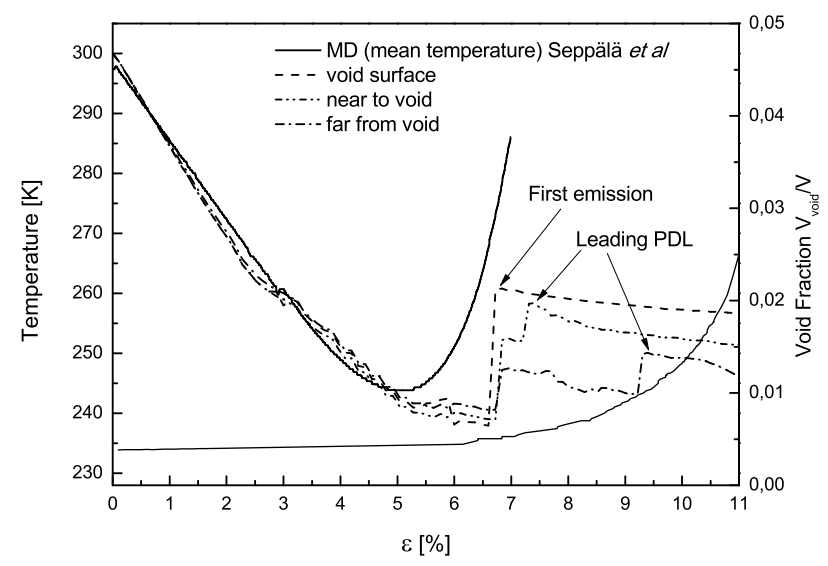

Figure 3.11: Temperature evolution during void growth at different positions within the sample: void surface, position near the void surface and position far from the void surface $\left(T_{0}=300 \mathrm{~K}\right.$ and $\left.\dot{\epsilon}=10^{10} \mathrm{~s}^{-1}\right)$.

tween both curves appear modest within the range of the plot, the discrepancy between the stacking-fault energies predicted by both potentials is significant. Thus, whereas, as already noted, the EAM-Mishin potential predicts an accurate stacking-fault energy of $44.6 \mathrm{~mJ} / \mathrm{m}^{2}$, the EAM-Johnson potential predicts a much higher value of $98 \mathrm{~mJ} / \mathrm{m}^{2}$ ). Given the importance of Shockley partials as fundamental mechanisms of deformation during nanovoid growth in copper, from these discrepancies we may expect vastly differing predictions of dislocation structure to result from the potentials, with the EAM-Mishin predictions presumably the most accurate of the two.

We begin by comparing the virial stress and void volume fraction curves vs engineering strain simulated with HotQC using both potentials. The triaxial expansion is carried out at $T_{0}=300 \mathrm{~K}$ and relatively low deformation rate $\dot{\varepsilon}=10^{7} \mathrm{~s}^{-1}$. It might be observed that the first set of curves in Fig. 3.16 show a different evolution, with the EAM-Johnson curve exhibiting stiffer behavior in the plateau region. The reasons for this discrepancy may be ascertained by comparing the normalized void volume curves in Fig. 3.16 and the dislocation structures formed immediately before the first yield point (2.8\% and 3.2\% strain for EAM-Mishin and EAM-Johnson results, respectively) and at the beginning of the virial stress drop $(6.4 \%$ and $6.8 \%$ strain for EAM-Mishin and EAM-Johnson results, respectively).

The differences between the two potentials are most striking as regards the predicted dislocation structures and their evolution. Fig. 3.17 depicts the 


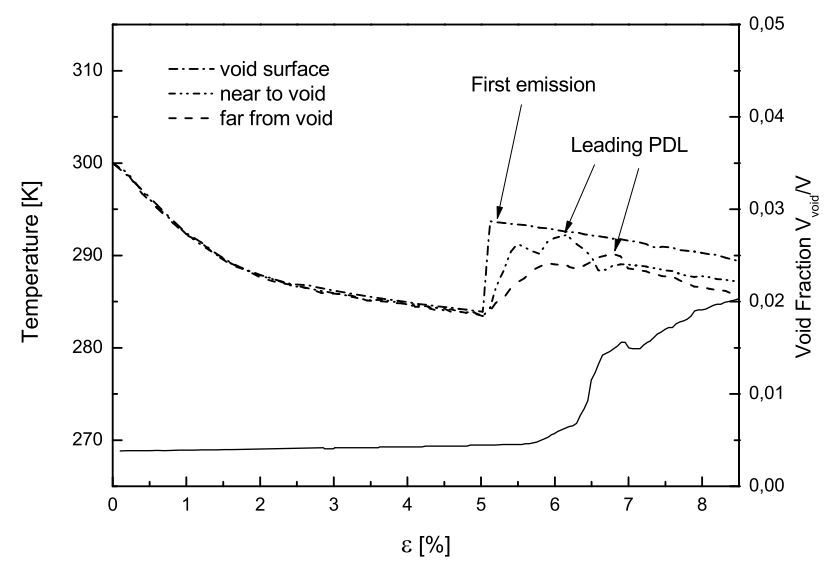

Figure 3.12: Temperature evolution during void growth at different positions within the sample: void surface, position near the void surface and position far from the void surface $\left(T_{0}=300 \mathrm{~K}\right.$ and $\left.\dot{\epsilon}=10^{8} \mathrm{~s}^{-1}\right)$.

dislocation structures formed immediately before the first yield point, at $2.8 \%$ and $3.2 \%$ strain for EAM-Mishin and EAM-Johnson, respectively. As can be seen in the figure, both potentials predict an octahedral void shape, which differ in slight detail only. By contrast, the subsequent predicted void growth differs sharply between the two potentials. Thus, as already discussed, at the critical strain of $\epsilon=6.4 \%$ the EAM-Mishin potential predicts the emission of prismatic dislocation loops (PDLs) from the void, Fig. 3.18a, in agreement with previous studies (Tsuru and Shibutani, 2007; Seppälä et al., 2004a). By contrast, in the case of the EAM-Johnson the octahedral dislocation structure is considerably more stable and becomes locked in, Fig. 3.18b, thereby greatly retarding the growth of the nanovoid. These vastly differing behaviors serve as stark reminder of the strong dependence of the fine details of the predicted microstructures on the choice of interatomic potential.

\section{Summary and concluding remarks}

We have presented a study of dynamic nanovoid growth in copper single crystals under prescribed to volumetric strain rates ranging from from moderate $\left(\dot{\epsilon}=10^{5} \mathrm{~s}^{-1}\right)$ to high $\left(\dot{\epsilon}=10^{10} \mathrm{~s}^{-1}\right)$. The study extends downward the range of strain rates previously studied by means of molecular dynamics (Seppälä et al., 2004a; Dávila et al., 2005; Bringa et al., 2010b). We recall, by way of putting the range of strain rates under consideration in perspec- 


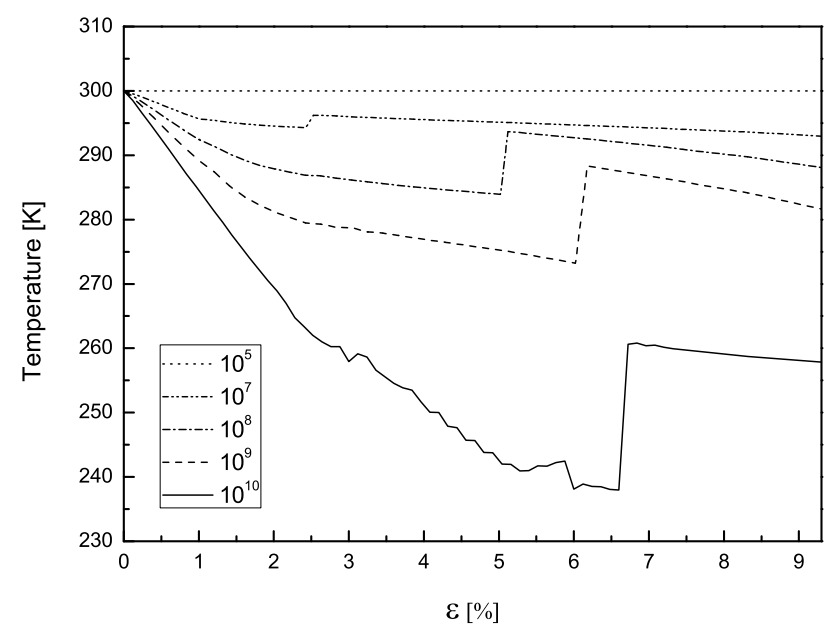

Figure 3.13: Temperature evolution versus strain at strain rates ranging from $10^{5}$ to $10^{10}$ $\mathrm{s}^{-1}$ at the void surface.

tive, that, in dynamic testing of materials, strain rates of $10^{7} \mathrm{~s}^{-1}$ and higher are considered exceedingly high and a rarely achieved in engineering applications. Thus, typical servo-hydraulic testing machines deliver strain rates in the order of $10^{0} \mathrm{~s}^{-1}$ whereas Hopkinson-bar tests deliver strain rates of the order of $10^{4} \mathrm{~s}^{-1}$. Higher strain rates require highly-engineered test configurations such as plate-impact experiments (Abou-sayed et al., 1976; Clifton, 1987), which deliver strain rates of up to $10^{7} \mathrm{~s}^{-1}$. Strain-rates in excess of this value are achieved rarely in practice and only under extreme conditions of loading and deformation. Therefore, the ability to elucidate material behavior at moderate strain rates below $10^{7} \mathrm{~s}^{-1}$ is essential to making contact with material testing and engineering applications.

We gain access to the lower strain rates by accounting for thermal vibrations in an entropic sense within the framework of maximum-entropy non-equilibrium statistical mechanics. This statistical treatment eliminates the need to track each thermal vibration of each individual atom in the sample, as required by molecular dynamics. We additionally account for heat conduction by means of empirical atomic-level kinetic laws, which may be regarded as atomic-level analogs of Fourier's law of heat conduction. The resulting mean trajectories of the atoms are smooth and can be integrated implicitly using large time steps, greatly in excess of those required by molecular dynamics. We also gain access to large computational cells and, cor- 


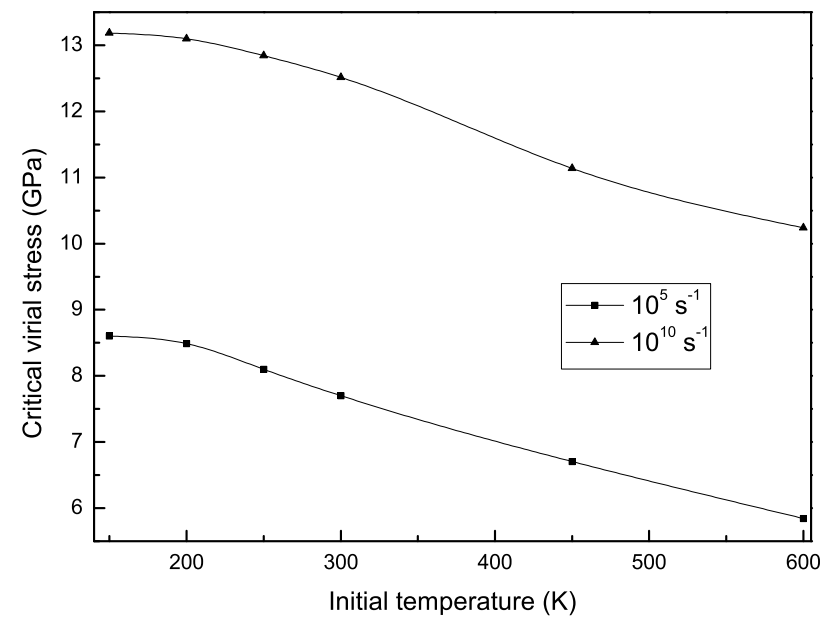

Figure 3.14: Computed critical virial stress (pressure) versus temperature in the range of 150 to $600 \mathrm{~K}$ at low and high strain rates, $10^{5} \mathrm{~s}^{-1}$ and $10^{10} \mathrm{~s}^{-1}$, respectively.

respondingly, physically realistic nanovoid densitities, by means of spatial coarse-graining using the quasicontinuum method (Tadmor et al., 1996).

On this basis, we identify a transition, somewhere between $10^{7}$ and $10^{8} \mathrm{~s}^{-1}$, between two regimes: a quasistatic regime characterized by nearly isothermal behavior and low dislocation velocities; and a dynamic regime characterized by nearly adiabatic conditions and high dislocation velocities. We also elucidate the precise mechanisms underlying dislocation emission from the nanovoids during cavitation. We find that, at the onset of cavitation, leading Shockley partial dislocations are emitted from the void surface, which subsequently glide and combine with dislocations moving on another intersecting $\{111\}$ planes. These reactions result in the formation of Lomer-Cottrell dislocations along $\langle 110\rangle$ directions and an octahedral arrangement of the surface atoms. This octahedral structure is very stable and temporarily locks the movement of the leading Shockley partials. As the applied strain increases, secondary shear loops grow on $\{111\}$ planes and along the edges of the octahedron. These additional Shockley partial dislocations are highly mobile and glide away from the void. The $1 / 6\langle\overline{11} 0\rangle$ stair-rod sessile dislocations along the intersection of pairs of $\{111\}$ planes form a closed loop with rhomboid shape and centered on $\langle 110\rangle$ directions, which provides the basis for the subsequent emission of prismatic dislocation loops away from the void. These structures and their evolution are consistent with the findings of previous studies (Hirth and Lothe, 1991; Hull and Bacon, 2001; Bulatov et al., 2010; 


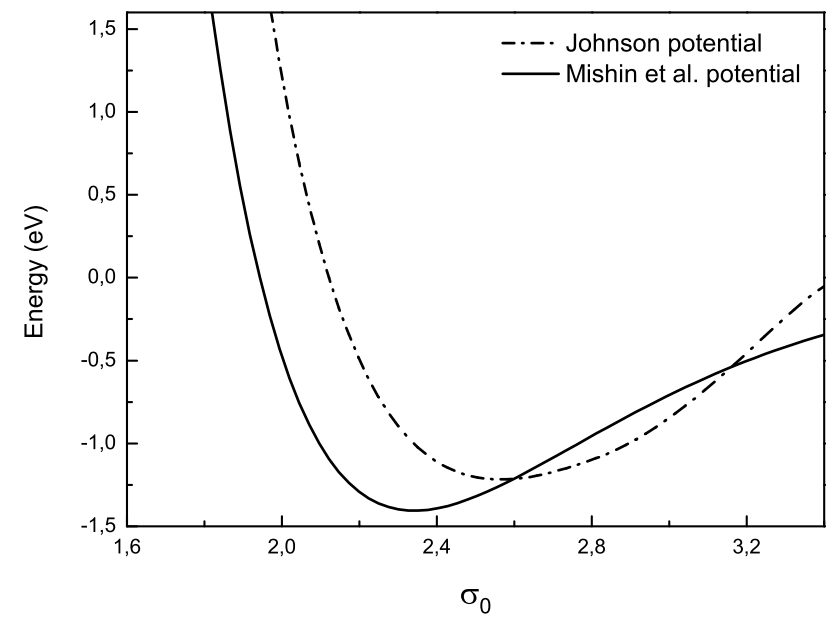

Figure 3.15: Total internal energy as a function of the distance between atoms for copper provided by the interatomic potentials by Johnson (1988) and Mishin et al. (2001).

Bringa et al., 2010a; Rudd, 2009) for crystals of different structures.

We additionally investigate the sensitivity of the results of the analysis to the choice of interatomic potential by comparing two EAM-type potentials by Mishin et al. (2001) (EAM-Mishin) and Johnson (1988) (EAM-Johnson). The differences between the two potentials are most striking as regards the predicted dislocation structures and their evolution. Thus, both potentials predict an octahedral void shape following the onset of cavitation. By contrast, the subsequent predicted void growth differs sharply between the two potentials. Thus, the EAM-Mishin potential predicts the emission of prismatic dislocation loops (PDLs) from the void, in agreement with the findings of previous studies (Tsuru and Shibutani, 2007; Seppälä et al., 2004a). By contrast, the EAM-Johnson the octahedral dislocation structure is considerably more stable and becomes locked in, thereby greatly retarding the growth of the nanovoid. Confidence in the EAM-Mishin potential is further buoyed by the observation that the EAM-Mishin potential predicts an accurate stacking-fault energy of $44.6 \mathrm{~mJ} / \mathrm{m}^{2}$, whereas the EAM-Johnson potential predicts a much higher value of $98 \mathrm{~mJ} / \mathrm{m}^{2}$. This sensitive dependence of the fine details of the predicted microstructures on the choice of interatomic potential underscores the need for accurate interatomic potentials which, in particular, match key material properties such as stacking-fault energies.

Similar concerns regarding model fidelity arise as regards the treatment 


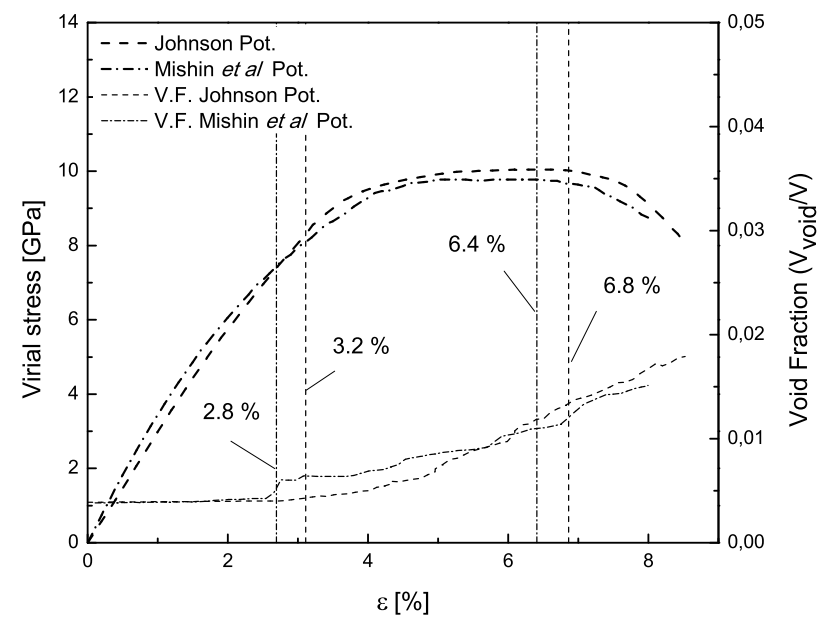

Figure 3.16: Comparison of virial stress and void volume fraction vs engineering strain simulated with HotQC using two potentials: EAM-Johnson (Johnson, 1988) and EAMMishin (Mishin et al., 2001). The triaxial expansion is carried out at $T_{0}=300 \mathrm{~K}$ and $\dot{\varepsilon}=10^{7} \mathrm{~s}^{-1}$.

of transport properties. Thus, in the present study we account for heat conduction by means of an empirical atomic-level heat equation, cf. 2.3. In particular, transport properties are not predicted implicitly by the model, as in the case of molecular dynamics, but are directly input into the model. This treatment of transport properties might appear to add to the empiricismand detract from the predictiveness - of the calculations. However, it should be noted that the resulting kinetic laws are empirical - and, therefore, subject to modeling - in exactly the same manner as the conventional interatomic potentials used in molecular dynamics are themselves empirical. In this sense, the use of empirical transport equations does not add significantly to the level of empiricism inherent to molecular dynamics. In addition, the transport properties that are implicitly predicted by molecular dynamics are often grossly inaccurate, as small errors in activation energies translate into exceedingly large errors in transport rates, and as major contributions to transport, such as electronic conductivity in metals, may be entirely unaccounted for by the interatomic potentials. In these cases, the ability to input measuredand therefore realistic - transport properties into the calculations may indeed be regarded as an advantage and not as a disadvantage.

The HotQC statistical treatment of thermal vibrations, upled to atomic- 


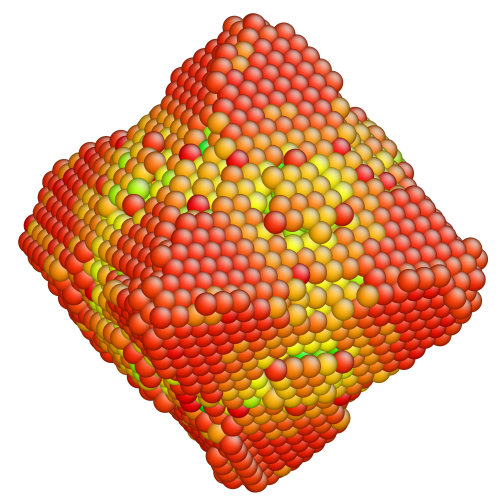

(a)

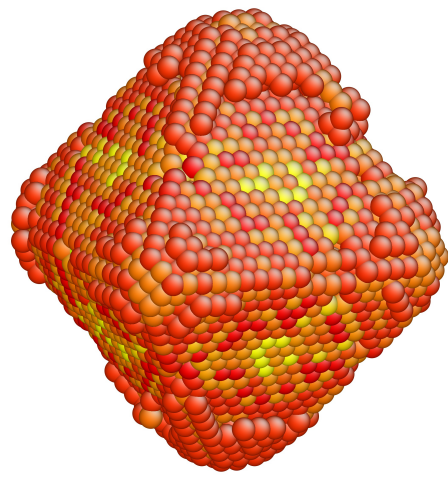

(b)

Figure 3.17: Octahedral shape of the atoms encompassing the surface of the void immediately before the first yield point, obtained using the quasicontinuum version of HotQC (a) at $\varepsilon=2.8 \%$ for simulation using the EAM-Mishin potential, and (b) at $\varepsilon=3.2 \%$ for simulation using the EAM-Johnson potential.

level heat conduction, has a number of additional far-reaching consequences. Thus, this treatment results in mean trajectories of the particles that are smooth on the time scale of thermal vibrations. This smooth mesoscopic dynamics can then be integrated implicitly using time steps much larger than those required by molecular dynamics. In this sense, we may regard HotQC as a method of temporal coarse-graining and as a multiple time-scale method, combining an effective treatment of thermal vibrations with a direct treatment of mesoscopic dynamics. The smoothness of the mesoscopic HotQC trajectories additional opens the way to spatial coarse-graining, e. g., by recourse to the quasi-continuum method. Thus, naïve dynamic extensions of the quasicontinuum based on a direct Galerkin reduction of the equations of motion suffer from the well-known shortcoming of spurious internal reflections of short wavelength, high-frequency, phonons off of spatially coarse-grained regions. These spurious internal reflections in turn result in phonon trapping - and excessive heating-in finely-resolved regions. Through the elimination of thermal vibrations from the dynamics, such spurious internal reflections are likewise eliminated or, at least, mitigated, thus effectively resolving a long-standing open issue with dynamic quasicontinuum. 


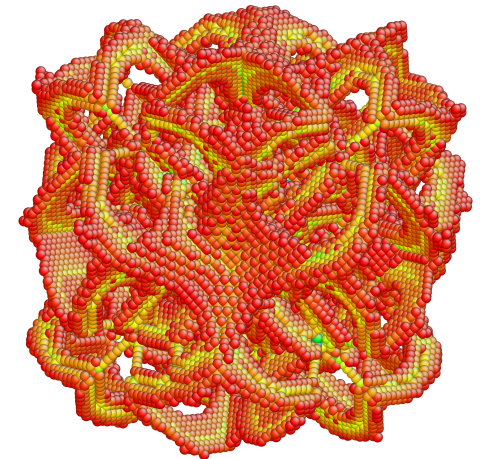

(a)

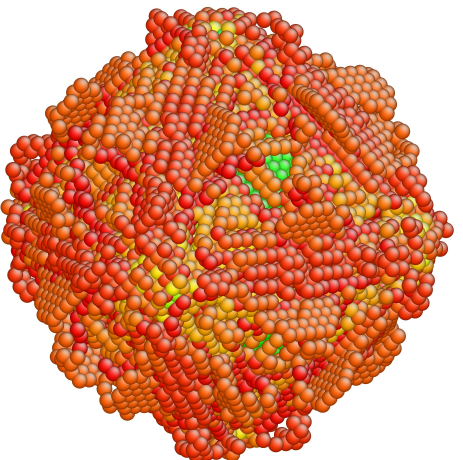

(b)

Figure 3.18: Dislocation structures around the surface of the void and the beginning of the virial stress drop, obtained using the quasicontinuum version of HotQC (a) at $\varepsilon=6.4 \%$ for simulation using the EAM-Mishin potential, and (b) at $\varepsilon=6.8 \%$ for simulation using the EAM-Johnson potential.

\section{Acknowledgements}

We gratefully acknowledge the support of the Ministerio de Ciencia e Innovación of Spain (DPI2009-14305-C02-01 and DPI2012-32508) and from the U. S. Army Research Laboratory (ARL) through the Materials in Extreme Dynamic Environments (MEDE) Collaborative Research Alliance (CRA) under Award Number W911NF-11-R-0001. P.A. and M.O. gratefully acknowledge support from the Department of Energy National Nuclear Security Administration under Award Number DE-FC52-08NA28613 through Caltech's ASC/PSAAP Center for the Predictive Modeling and Simulation of High Energy Density Dynamic Response of Materials.

Abou-sayed, A. S., Clifton, R. J., Hermann, L., 1976. Oblique-plate impact experiment. Experimental Mechanics 16 (4), 127-132.

Andersen, H. C., 1980. Molecular dynamics simulations at constant pressure and/or temperature. The Journal of Chemical Physics 72 (4), 2384-2393.

Ariza, M. P., Romero, I., Ponga, M., Ortiz, M., 2012. Hotqc simulation of nanovoid growth under tension in copper. International Journal of Fracture $174,75-85$. 
Belak, J. F., 2002a. Multi-scale applications to high strain-rate dynamic fracture. Journal of Computer Aided-Materials Design 9, 165-172.

Belak, J. F., 2002b. Multi-scale applications to high strain-rate dynamic fracture. Journal of Computer-Aided Materials Design 9, 165-172.

Bringa, E., Lubarda, V., Meyers, M., 2010a. Response to shear impossibilitycomments on void growth by dislocation emission and void growth in metals. Scripta Materialia 63 (1), $148-150$.

Bringa, E. M., Traiviratana, S., Meyers, M. A., 2010b. Void initiation in fcc metals: Effect of loading orientation and nanocrystalline effects. Acta Materialia 58 (13), 4458-4477.

Bulatov, V. V., Wolferm, W. G., Kumar, M., 2010. Shear impossibility: Comments on void growth by dislocation emission and void growth in metals: Atomistic calculations. Scripta Materialia 63 (1), $144-147$.

Bussi, G., Parrinello, M., 2008. Stochastic thermostats: comparison of local and global schemes. Computer Physics Communications 179 (13), 26 - 29.

Callen, H., 1985. Thermodynamics and an Introduction to Thermostatistics. John Wiley \& Sons.

Carter, C. B., Ray, I. L. F., 1977. On the stacking-fault energies of copper alloys. Philosophical Magazine 35 (1), 189-200.

Clifton, R. J., 1987. Plastic-deformation of metals at strain rates of 10-5S-1 to $10-7 \mathrm{~S}-1$ by means of pressure-shear plate impact experiments. Journal of Metals 39 (10), A52.

Coleman, B. D., Noll, W., 1963. The thermodynamics of elastic materials with heat conduction and viscosity. Archive for Rational Mechanics and Analysis 13 (3), 167-178.

Dávila, L. P., Erhart, P., Bringa, E. M., Meyers, M. A., Lubarda, V. A., Schneider, M. S., Becker, R., Kumar, M., 2005. Atomistic modeling of shock-induced void collapse in copper. Applied Physics Letters 86, 161902.

De Groot, S. R., Mazur, P., 1962. Non-equilibrium thermodynamics. NorthHolland, Amsterdam. 
Germann, T. C., Holian, B. L., Kadau, K., Lomdahl, P. S., 2005. Multibillionatom molecular dynamics simulations of shockwave phenomena on BlueGene/L. In: Simos, T and Maroulis, G (Ed.), Advances in Computational Methods in Sciences and Engineering 2005, Vols 4 A \& 4 B. Vol. 4A-4B of LECTURE SERIES ON COMPUTER AND COMPUTATIONAL SCIENCES. Amer Chem Soc; Amer Phys Soc, pp. 1138-1141.

Girifalco, L. A., 2000. Statistical Mechanics of Solids. Oxford University Press.

Heller, A., 2002. How metals fails. Science and Technology Review, 13-20.

Hill, T., 1987. Statistical Mechanics: Principles and Selected Applications. Dover Publications.

Hirth, J. P., Lothe, J., 1991. Theory of Dislocations, 2nd New edition of Revised edition. Krieger Publishing Company.

Honeycutt, J. D., Andersen, H. C., 1987. Molecular dynamics study of melting and freezing of small lennard-jones clusters. The Journal of Physical Chemistry 91 (19), 4950-4963.

Hoover, W. G., Mar 1985. Canonical dynamics: Equilibrium phase-space distributions. Phys. Rev. A 31, 1695-1697.

Hughes, T. J. R., 2000. The finite element method: Linear static and dynamic finite element analysis. Dover.

Hull, D., Bacon, D. J., 2001. Introduction to dislocations. ButterworthHeinemann.

Hünenberger, P., 2005. Thermostat algorithms for molecular dynamics simulations. In: Holm, C., Kremer, K. (Eds.), Advanced Computer Simulation. Vol. 173 of Advances in Polymer Science. pp. 105-149.

Jaynes, E. T., 1957a. Information theory and statistical mechanics i. Physical Review Series II 106 (4), 620-630.

Jaynes, E. T., 1957b. Information theory and statistical mechanics ii. Physical Review Series II 108 (2), 171-190. 
Johnson, R. A., 1988. Analytic nearest-neighbor model for fcc metals. Physical Review B 37, 3924-3931.

Knap, J., Ortiz, M., 2001. An analysis of the quasicontinuum method. Journal of the Mechanics and Physics of Solids 49, 1899-1923.

Kulkarni, Y., Knap, J., Ortiz, M., 2008. A variational approach to coarse graining of equilibrium and non-equilibrium atomistic description at finite temperature. Journal of the Mechanics and Physics of Solids 56, 1417-1449.

Lubarda, V. A., 2011. Emission of dislocations from nanovoids under combined loading. International Journal of Plasticity 27 (2), 181 - 200.

Marian, J., Knap, J., Ortiz, M., 2004. Nanovoid cavitation by dislocation emission in aluminum. Physical Review Letters 93 (16).

Marian, J., Knap, J., Ortiz, M., 2005. Nanovoid deformation in aluminum under simple shear. Acta Materialia 53 (10), 2893 - 2900.

Meyers, M. A., Traiviratana, S., Lubarda, V. A., Benson, D. J., Bringa, E. M., 2009. The role of dislocations in the growth of nanosized voids in ductile failure of metals. JOM Journal of the Minerals, Metals and Materials Society 61, 35-41.

Miller, R. E., Tadmor, E. B., 2002. The quasicontinuum method: Overview, applications and current directions. Journal of Computer-Aided Materials Design 9, 203-239.

Mishin, Y., Mehl, M., Papaconstantopoulos, D., Voter, A., Kress, J., 2001. Structural stability and lattice defects in copper: Ab initio, tight-binding, and embedded-atom calculations. Physical Review B 63 (22).

Newmark, N. M., 1959. A Method of Computation for Structural Dynamics. Journal of the Engineering Mechanics Division, ASCE 85, 2094.

Nosé, S., 1984. A unified formulation of the constant temperature molecular dynamics methods. The Journal of Chemical Physics 81 (1), 511-519.

Oakley, D. R., Knight, N. F., 1995. Adaptive dynamic relaxation algorithm for non-linear hyperelastic structures part i. formulation. Computer Methods in Applied Mechanics and Engineering 126 (1-2), 67 - 89. 
Ogata, S., Li, J., Yip, S., 2002. Ideal pure shear strength of aluminum and copper. Science 25 (298), 807-811.

Onsager, L., 1931a. Reciprocal relations in irreversible processes. i. Physical Review 37 (4), 405-426.

Onsager, L., 1931b. Reciprocal relations in irreversible processes. ii. Physical Review 38 (12), 2265-2279.

Ortiz, M., Molinari, A., 1992. Effect of strain-hardening and rate sensitivity on the dynamic growth of a void in plastic material. ASME J. Appl. Mech 59 (1), 48-53.

Ponga, M., Romero, I., Ortiz, M., Ariza, M. P., 2012. Finite temperature nanovoids evolution in fcc metals using quasicontinuum method. Key Engineering Materials 488-489, 387-390.

Reina, C., Marian, J., Ortiz, M., 2011. Nanovoid nucleation by vacancy aggregation and vacancy-cluster coarsening in high-purity metallic single crystals. Phys. Rev. B 84, 104117.

Rudd, R. E., 2009. Void growth in bcc metals simulated with molecular dynamics using the Finnis-Sinclair potential. Philosophical Magazine 89 (3436), 3133-3161.

Seppälä, E. T., Belak, J., Rudd, R. E., 2004a. Effect of stress triaxiality on void growth in dynamic fracture of metals: A molecular dynamics study. Physical Review B 69 (13), 134101.

Seppälä, E. T., Belak, J. F., Rudd, R. E., 2004b. Effect of stress triaxiality on void growth in dynamic fracture of metals: A molecular dynamics study. Physical Review B 69 (13), 134101.

Seppälä, E. T., Belak, J. F., Rudd, R. E., 2004. Effect of stress triaxiality on void growth in dynamic fracture of metals: A molecular dynamics study. Physical Review B 69, 134101.

Seppälä, E. T., Belak, J. F., Rudd, R. E., 2005. Three-dimensional molecular dynamics simulations of void coalescence during dynamic fracture of ductile metals. Physical review B 71 (6). 
Stevens, A. L., Davison, L., Warren, W. E., 1972. Spall fracture in aluminum monocrystals - Dislocation-dynamics approach. Journal of Applied Physics 43 (12), 4922-4927.

Stukowski, A., Albe, K., 2010. Extracting dislocations and non-dislocation crystal defects from atomistic simulation data. Modelling and Simulation in Materials Science and Engineering 18 (8), 085001.

Tadmor, E. B., Ortiz, M., Phillips, R., 1996. Quasicontinuum analysis of defects in solids. Philosophical Magazine 73, 1529-1563.

Traiviratana, S., Bringa, E., J.B., D., et al., 2007. Void growth in single and bicrystalline metals: Atomistic calculations. Shock Compression of Condensed Matter 955 (1), 343-346.

Tsuru, T., Shibutani, Y., 2007. Initial yield process around a spherical inclusion in single-crystalline aluminium. Journal of Physics D: Applied Physics $40(7), 2183$.

Tsuzuki, H., Branicio, P. S., Rino, J. P., 2008. Accelerating dislocations to transonic and supersonic speeds in anisotropic metals. Applied Physics Letters 92 (19).

Underwood, P., 1983. Dynamic relaxation. In: Computational Methods for Transient Dynamic Analysis. North Holland.

Venturini, G., Wang, K., Romero, I., Ariza, M. P., Ortiz, M., 2014. Atomistic Long-Term Simulation of Heat and Mass Transport. J. Mech. Phys. Solids $73,242-268$.

Vetterling, W., Press, W., Flannery, B., Teukolsky, S., Press, W. H., 2002. Numerical Recipes Example Book $\mathrm{C}++$. Cambridge University Press.

Yang, Q., Stainier, L., Ortiz, M., 2006. A Variational Formulation of the Coupled Thermo-Mechanical Boundary-Value Problem for General Dissipative Solids. Journal of the Mechanics and Physics of Solids 54 (2), 401-424.

Zubarev, D., 1974. Nonequilibrium Statistical Thermodynamics. Consultants Bureau - New York. 